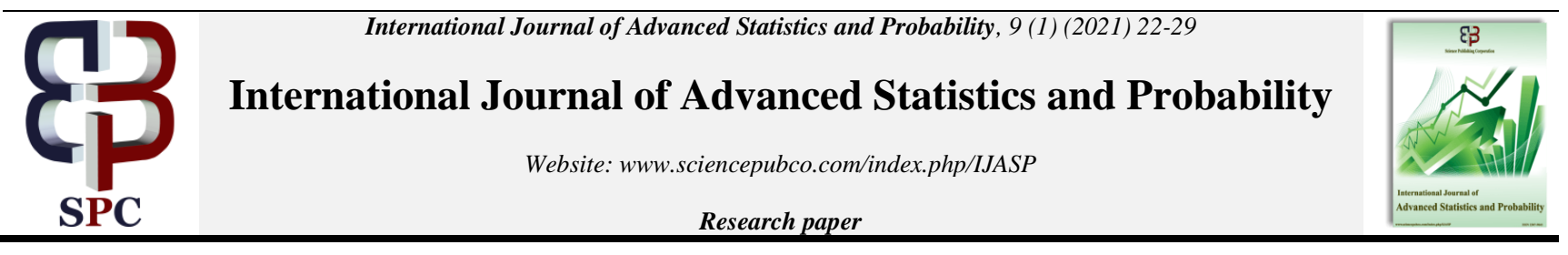

\title{
A new generalized transmuted weibull distribution: properties and applications
}

\author{
Elebe Emmanuel Nwezza ${ }^{1} *$, Uchenna U. Uwadi ${ }^{2}$, Chukwunenye I. Okonkwo ${ }^{3}$ \\ ${ }^{1}$ Department of Mathematics and Statistics, Alex Ekwueme Federal University Ndufu-Alike, Ikwo, Nigeria \\ *Corresponding author E-mail: elebe.nwezza@funai.edu.ng
}

\begin{abstract}
We introduce a new generalized transmuted-Weibull distribution and studied some of the mathematical characterizations of the new distribution. The quantile function, entropy, moment, moment generating function, and order statistics of the distribution are derived. The unknown parameters of the distribution were estimated through the maximum likelihood approach. The potential and flexibility of the new generalized transmuted-Weibull are illustrated by comparing it with other known distribution using real-life data sets.
\end{abstract}

Keywords: Another Generalized Transmuted-G; Weibull Distribution; Entropy; Moments; Maximum Likelihood.

\section{Introduction}

The Weibull distribution has gained a wide range of applicability in modelling data in reliability, engineering, biological, and risk studies It has the potential to model constant, monotone increasing and decreasing hazard rates but not non-monotone hazard rates. The addition of parameter(s) to already existing (classical) distributions to generate family of distributions extends and provides more flexibility to the classical distributions in modelling data which ordinarily would have not provided a good fit. There are many extension of classical Weibull distribution in the statistical literatures including Exponentiated-Weibull distribution by Mudholkar and Srivastava[1], Exponentiated Weibull family by [2], A Generalized Weibull family by [3], Marshall Olkin extended Weibull by [4], Beta-Weibull by [5], Beta modified Weibull by [6], KumaraswamyWeibull by [7] and Transmuted -Weibull by Aryal and Tsokos [8], Gumbel-Weibull by AlAqtash et al. [9] For more on Weibull extension see [10].

The main motivations of this paper are to propose a more flexible extension of Weibull distribution that can model bathtub, reversedbathtub, unimodal, increasing and decreasing hazard rate shapes.

The remainder of the paper is organised as follows; Section 2, introduced the new distribution and its sub-models. In Section 3, the mathematical properties including the mode, quantile function, moments, moment generating function, and the Renyi entropy are presented. The ordered statistics of the new distribution was considered in Section 4, while in Sections 5 and 6, the method of parameter estimation and applications of the distribution on two real-life data sets were presented.

\section{Materials and methods}

Merovci et al. [11] defined the cumulative density function (cdf) of Another Generalized Transmuted family of distributions as

$$
\mathrm{F}(\mathrm{x})=(1+\lambda)\left[1-(\overline{\mathrm{G}}(\mathrm{x}))^{\alpha}\right]-\lambda\left[1-(\overline{\mathrm{G}}(\mathrm{x}))^{\alpha}\right]^{2}
$$

With associated probability density function (pdf)

$$
\mathrm{f}(\mathrm{x})=\alpha \mathrm{g}(\mathrm{x})(\overline{\mathrm{G}}(\mathrm{x}))^{\alpha-1}\left\{1+\lambda-2 \lambda\left[1-(\overline{\mathrm{G}}(\mathrm{x}))^{\alpha}\right]\right\}
$$

Where $\alpha>0$ and $|\lambda| \leq 1$ are the additional shape parameters, and $\bar{G}(x)=1-G(x)$ is the survival function (sf) of the baseline distribution. According to [12], the cdf of a random variable, $X$, having Weibull distribution with zero location parameter is defined by

$$
\mathrm{G}(\mathrm{x})=1-\exp \left[-\left(\frac{\mathrm{x}}{\beta}\right)^{\theta}\right] \text {. }
$$

The associated sf and pdf are, respectively, given by 
$1-G(x)=\exp \left[-\left(\frac{x}{\beta}\right)^{\theta}\right]$

and

$g(x)=\frac{\theta}{\beta}\left(\frac{x}{\beta}\right)^{\theta-1} \exp \left[-\left(\frac{x}{\beta}\right)^{\theta}\right]$

Using equation (1), we define the cdf of the AGT-W distribution as

$\mathrm{F}(\mathrm{x})=(1+\lambda)\left\{1-\exp \left[-\alpha\left(\frac{\mathrm{x}}{\beta}\right)^{\theta}\right]\right\}-\lambda\left\{1-\exp \left[-\alpha\left(\frac{\mathrm{x}}{\beta}\right)^{\theta}\right]\right\}^{2}$

with the corresponding density function and hazard rate function (hrf)

$\mathrm{f}(\mathrm{x})=\frac{\alpha \theta}{\beta^{\theta}} \mathrm{x}^{\theta-1} \exp \left[-\alpha\left(\frac{\mathrm{x}}{\beta}\right)^{\theta}\right]\left\{1+\lambda-2 \lambda \exp \left[-\alpha\left(\frac{\mathrm{x}}{\beta}\right)^{\theta}\right]\right\}$

and

$\mathrm{hrf}=\frac{\alpha \theta \mathrm{x}^{\theta-1} \exp \left[-\alpha\left(\frac{\mathrm{x}}{\beta}\right)^{\theta}\right]\left\{1+\lambda-2 \lambda \exp \left[-\alpha\left(\frac{\mathrm{x}}{\beta}\right)^{\theta}\right]\right\}}{\beta^{\theta}\left[1-\left((1+\lambda)\left\{1-\exp \left[-\alpha\left(\frac{\mathrm{x}}{\beta}\right)^{\theta}\right]\right\}-\lambda\left\{1-\exp \left[-\alpha\left(\frac{\mathrm{x}}{\beta}\right)^{\theta}\right]\right\}^{2}\right)\right]}$

\subsection{Submodels of AGT-W distribution}

The AGT-W generalizes many distribution including

i) At $\lambda=0$ (7) reduces to three-parameter exponentiated Weibull (E-W) distribution due to Mudholka et al. [2]

ii) At $\lambda=0$ and $\alpha=1$ (7) reduces to two-parameter Weibull (W) distribution defined by Johnson et al. [12].

iii) At $\alpha=1$ (7) reduces to transmuted-Weibull (T-W) due to Aryal and Tsokos [8].

\subsection{Shapes of AGT-W density and hazard rate functions}

The possible shapes of AGT-W density and hazard rate functions for some selected parameter values are, respectively, shown in Figure 1 and Figure 2.

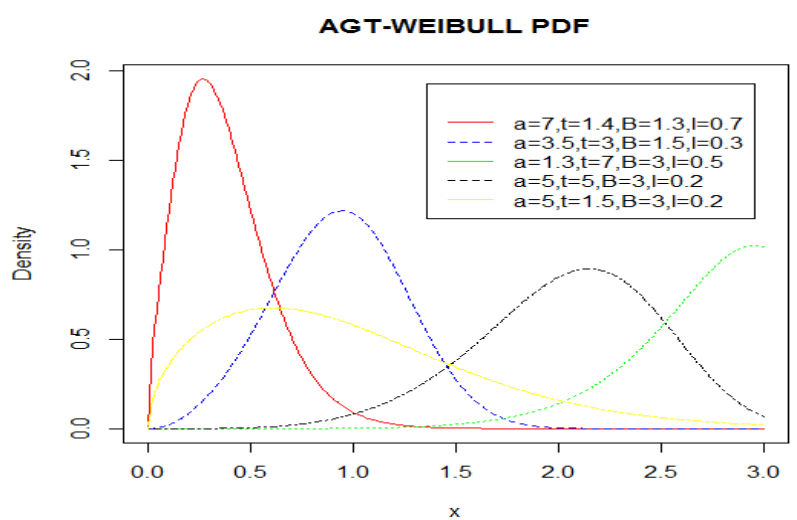

Fig. 1: Plots of AGT-W Density Function at Various Parameter Values.

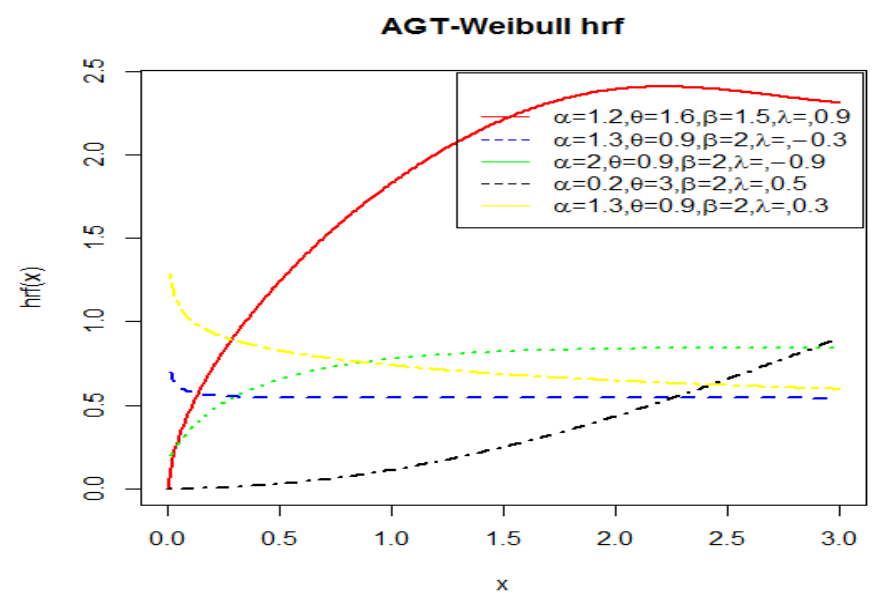

Fig. 2: Plots of AGT-W HRF at Different Values of the Parameters. 


\subsection{Mode of AGT-W distribution}

The mode of AGT-W distribution can be obtained numerically as critical points of equation (9) which is obtained by taking the derivative of logarithm of equation (7) with respect to (w.r.t.) $x$.

$$
\frac{\theta-1}{x}-\frac{\theta \alpha x^{\theta-1}}{\beta^{\theta}}+\frac{2 \lambda \alpha \theta x^{\theta-1} \exp \left[-\alpha\left(\frac{x}{\beta}\right)^{\theta}\right]}{\beta^{\theta}\left\{1+\lambda-2 \lambda \exp \left[-\alpha\left(\frac{x}{\beta}\right)^{\theta}\right]\right\}}=0
$$

Equation (9) indicates that there may be more than one root to the equation and the second derivative of logarithm of equation (7) is given below

$$
\begin{aligned}
& \psi(x)=\left(\left\{1+\lambda-2 \lambda \exp \left[-\alpha\left(\frac{x}{\beta}\right)^{\theta}\right]\right\}\left[\beta^{\theta}(\theta-1)-\alpha \theta x^{\theta}\right]-2 \lambda \alpha \theta x^{\theta} \exp \left[-\alpha\left(\frac{x}{\beta}\right)^{\theta}\right]\right) \times \\
& \frac{x^{\theta-1} \exp \left[-\alpha\left(\frac{x}{\beta}\right)^{\theta}\right]}{\beta^{2 \theta}\left[1+\lambda-2 \lambda \exp \left[-\alpha\left(\frac{x}{\beta}\right)^{\theta}\right]\right]}+\frac{\theta-1}{x^{2}}-\frac{\theta(\theta-1) \alpha x^{\theta-2}}{\beta^{\theta}}=0 ;
\end{aligned}
$$

where

$\psi(x)=\frac{\partial^{2} \log f(x)}{\partial x^{2}}$

If $x=x_{0}$ is a root of the first derivative, the it corresponds to a local maximum if $\psi(x)>0$ for all $x<x_{0}$ and $\psi(x)<0$ for all $x>x_{0}$. It corresponds to local minimum if $\psi(x)<0$ for all $x<x_{0}$ and $\psi(x)>0$ for all $x>x_{0}$. It refers to an inflexion point if either $\psi(x)>0$ for all $x \neq x_{0}$ or $\psi(x)<0$ for all $x \neq x_{0}$.

\section{Moment}

Theorem: If $X$ follows AGT-W $(x, \alpha, \lambda, \theta, \beta)$, then the $r^{\text {th }}$ moment about the origin is obtained as

$$
E\left(X^{r}\right)=\beta^{r} \alpha^{-\frac{r}{\theta}} \Gamma\left(\frac{r}{\theta}+1\right)\left(1+\lambda-2^{-\frac{r}{\theta}} \lambda\right) .
$$

Proof:

By definition

$$
\begin{aligned}
E\left(X^{r}\right) & =\int_{0}^{\infty} x^{r} f(x) d x \\
& =\int_{0}^{\infty} x^{r}\left(\frac{\alpha \theta}{\beta^{\theta}} x^{\theta-1} \exp \left[-\alpha\left(\frac{x}{\beta}\right)^{\theta}\right]\left\{1+\lambda-2 \lambda \exp \left[-\alpha\left(\frac{x}{\beta}\right)^{\theta}\right]\right\}\right) d x
\end{aligned}
$$

Evaluating the integrals, we have

\begin{tabular}{|c|c|c|c|c|c|c|c|}
\hline$\alpha$ & $\theta$ & $\beta$ & $\lambda$ & $\mu_{1}^{\prime}$ & $\mu_{2}^{\prime}$ & $\mu_{3}^{\prime}$ & $\mu_{4}^{\prime}$ \\
\hline \multirow[t]{5}{*}{0.2} & 1.3 & 3 & -0.9 & 6.001 & 59.940 & 844.194 & 15663.6 \\
\hline & 3.6 & 2 & -0.5 & 2.571 & 7.3080 & 22.4612 & 73.627 \\
\hline & 2.3 & 2.3 & 0.1 & 4.208 & 21.329 & 123.052 & 784.227 \\
\hline & 2.8 & 1.4 & 0.3 & 2.360 & 6.300 & 18.387 & 57.601 \\
\hline & 5 & 2 & 0.9 & 2.828 & 8.228 & 24.524 & 74.691 \\
\hline \multirow[t]{4}{*}{0.7} & 1.3 & 3 & -0.9 & 2.289 & 8.723 & 46.870 & 331.77 \\
\hline & 3.6 & 2 & -0.5 & 1.815 & 3.643 & 7.907 & 18.302 \\
\hline & 2.3 & 2.3 & 0.1 & 2.441 & 7.175 & 24.012 & 88.764 \\
\hline & 5 & 2 & 0.9 & 2.201 & 4.985 & 11.565 & 27.417 \\
\hline 1.2 & 1.3 & 3 & -0.9 & 1.512 & 3.806 & 13.511 & 63.180 \\
\hline
\end{tabular}

$$
\begin{aligned}
E\left(X^{r}\right) & =(1+\lambda) \beta^{r} \alpha^{-\frac{r}{\theta}} \Gamma\left(\frac{r}{\theta}+1\right)-(2 \alpha)^{-\frac{r}{\theta}} \beta^{r} \lambda \Gamma\left(\frac{r}{\theta}+1\right) \\
& =\beta^{r} \alpha^{-\frac{r}{\theta}} \Gamma\left(\frac{r}{\theta}+1\right)\left[1+\lambda-2^{-\frac{r}{\theta}} \lambda\right] .
\end{aligned}
$$

We can obtain the numerical values of other characterizations which are function of the non-central moment. These characterizations include the Kurtosis denoted by $\gamma_{1}$, Skewness denoted by $\gamma_{2}$, and coefficient of variation denoted by $C V$ are, respectively, defined below

$$
\gamma_{1}=\frac{\mu_{4}^{\prime}-4 \mu_{3}^{\prime} \mu_{1}^{\prime}+6 \mu_{2}^{\prime} \mu_{1}^{\prime}-3 \mu_{1}^{\prime 4}}{\left(\mu_{2}^{\prime}-\mu_{1}^{\prime 2}\right)^{2}}, \gamma_{2}=\frac{\mu_{3}^{\prime}-3 \mu_{2}^{\prime} \mu_{1}^{\prime}+2 \mu_{1}^{\prime 3}}{\left(\mu_{2}^{\prime}-\mu_{1}^{\prime 2}\right)^{\frac{3}{2}}}, \text { and } C V=\sqrt{\frac{\mu_{2}^{\prime}}{\mu_{1}^{\prime 2}}-1}
$$

Table 1: The First Our Moments of AGT-W Distribution with Selected Parameter Values 


\begin{tabular}{|c|c|c|c|c|c|c|c|}
\hline & 3.6 & 2 & -0.5 & 1.563 & 2.700 & 5.046 & 10.056 \\
\hline & 2.3 & 2.3 & 0.1 & 1.931 & 4.490 & 11.888 & 34.764 \\
\hline & 2.8 & 1.4 & 0.3 & 1.244 & 1.751 & 2.696 & 4.454 \\
\hline & 5 & 2 & 0.9 & 1.976 & 4.018 & 8.369 & 17.813 \\
\hline \multicolumn{8}{|c|}{ Table 2: The First Our Moments of T-W Distribution with Selected Parameter Values } \\
\hline$\alpha \downarrow$ & $\theta$ & $\beta$ & $\lambda$ & $\mu_{1}^{\prime}$ & $\mu_{2}^{\prime}$ & $\mu_{3}^{\prime}$ & $\mu_{4}^{\prime}$ \\
\hline \multirow[t]{5}{*}{1} & 1.3 & 3 & -0.9 & 1.740 & 5.039 & 20.579 & 110.717 \\
\hline & 3.6 & 2 & -0.5 & 1.644 & 2.988 & 0.874 & 12.314 \\
\hline & 2.3 & 2.3 & 0.1 & 2.090 & 5.262 & 15.079 & 47.735 \\
\hline & 2.8 & 1.4 & 0.3 & 1.328 & 1.995 & 3.278 & 5.779 \\
\hline & 5 & 2 & 0.9 & 2.050 & 4.322 & 9.337 & 20.610 \\
\hline
\end{tabular}

Table 3: The First Our Moments of W Distribution with Selected Parameter Values

\begin{tabular}{|c|c|c|c|c|c|c|c|}
\hline$\alpha \downarrow$ & $\theta$ & $\beta$ & $\lambda$ & $\mu_{1}^{\prime}$ & $\mu_{2}^{\prime}$ & $\mu_{3}^{\prime}$ & $\mu_{4}^{\prime}$ \\
\hline 1 & 1.3 & 3 & 0 & 2.770 & 12.296 & 73.032 & 535.75 \\
\hline & 3.6 & 2 & & 1.801 & 3.557 & 7.525 & 16.834 \\
\hline & 2.3 & 2.3 & & 2.037 & 5.034 & 14.232 & 44.610 \\
\hline & 5 & 2 & & 1.836 & 3.549 & 7.148 & 14.902 \\
\hline
\end{tabular}

Table 4: The First Our Moments of E-W Distribution with Selected Parameter Values

\begin{tabular}{|c|c|c|c|c|c|c|c|}
\hline$\alpha$ & $\theta$ & $\beta$ & $\lambda$ & $\mu_{1}^{\prime}$ & $\mu_{2}^{\prime}$ & $\mu_{3}^{\prime}$ & $\mu_{4}^{\prime}$ \\
\hline \multirow[t]{4}{*}{0.2} & 1.3 & 3 & 0 & 9.556 & 146.258 & 2995.88 & 75794.7 \\
\hline & 3.6 & 2 & & 2.818 & 8.697 & 28.773 & 100.657 \\
\hline & 2.3 & 2.3 & & 4.102 & 20.405 & 116.14 & 732.892 \\
\hline & 5 & 2 & & 2.533 & 6.756 & 18.774 & 54.004 \\
\hline
\end{tabular}

The relationships between AGT-W and its sub-models are illustrated via the computation of moments about the origin. Table 1 shows the first four moments about the origin of AGT-W. Table 2 shows the first four moments about the origin of T-W ie $\mu_{A G T-W}^{\prime}=\mu_{T-W}^{\prime}$, $\forall \theta, \beta, \lambda$, when $\alpha=1$. However, Table 3 shows that of Weibull distribution ie $\mu_{A G T-W}^{\prime}=\mu_{W}^{\prime} \forall \theta$, $\beta$, when $\alpha=1$, and $\lambda=0$. Table 4 shows the first four moments about the origin of E-W ie $\mu_{A G T-W}^{\prime}=\mu_{E-W}^{\prime} \forall \alpha, \theta, \beta$, when $\lambda=0$. We observed that $\mu_{A G T-W}^{\prime}>\mu_{T-W}^{\prime}$, and $\mu_{A G T-W}^{\prime}>\mu_{W}^{\prime}$ when $\alpha<1 \forall \theta, \beta, \lambda$. Finally, $\mu_{A G T-W}^{\prime}<\mu_{E-W}^{\prime}$ when $\alpha>1 \forall \theta, \beta, \lambda$.

\subsection{Moment generating function (MGF)}

Theorem 2: Suppose $X$ follows AGT-W $(\theta, \alpha, \beta, \lambda)$, then the MGF of $X$ is given by

$M_{x}(t)=\sum_{r=0}^{\infty} \frac{t^{r}}{r !}\left\{\beta^{r} \alpha^{-\frac{r}{\theta}} \Gamma\left(\frac{r}{\theta}+1\right)\left[1+\lambda-2^{-\frac{r}{\theta}} \lambda\right]\right\}(11)$

Proof:

By definition

$$
\begin{aligned}
M_{x}(t) & =E\left(e^{t X}\right) \\
& =E\left(\sum_{r=0}^{\infty} \frac{(t x)^{r}}{r !}\right) \\
& =\sum_{r=0}^{\infty} \frac{t^{r}}{r !} E\left(X^{r}\right)
\end{aligned}
$$

Substituting the expression for $E\left(X^{r}\right)$ in equation (10) we have

$$
M_{x}(t)=\sum_{r=0}^{\infty} \frac{t^{r}}{r !}\left(\beta^{r} \alpha^{-\frac{r}{\theta}} \Gamma\left(\frac{r}{\theta}+1\right)\left[1+\lambda-2^{-\frac{r}{\theta}} \lambda\right]\right) .
$$

\section{Quantile function of AGT-W}

The quantile function corresponding toAGT-W $(\alpha, \theta, \beta, \lambda)$ distribution is the real solution of

$Q(u)=\beta\left[-\frac{1}{\alpha} \ln \left(\frac{\lambda-1+\sqrt{(1+\lambda)^{2}-4 \lambda u}}{2 \lambda}\right)\right]^{\frac{1}{\theta}} ;$

where $u$ is uniformly distributed within the interval $(0,1)$. By letting $u=0.5$ in (12), the median of AGT-W $(\alpha, \theta, \beta, \lambda)$ given by

$$
Q(0.5)=\beta\left[-\frac{1}{\alpha} \ln \left(\frac{\lambda-1+\sqrt{(1+\lambda)^{2}-2 \lambda}}{2 \lambda}\right)\right]^{\frac{1}{\theta}}
$$




\section{Renyi entropy}

The entropy of a random variable, $X$, with density function $f(x)$ is a measure of the variation of uncertainty and for any real parameter $\gamma>0$ and $\gamma \neq 1$, the Renyi entropy [13] is given by

$$
I_{R}(\gamma)=\frac{1}{1-\gamma} \log \left(\int_{-\infty}^{\infty} f^{\gamma}(x) d x\right)
$$

Theorem 3: Suppose random variable $X$ follows AGT-W $(\alpha, \theta, \beta, \lambda)$, then the Renyi entropy of $X$ is given by

$I_{R}(\gamma)=\frac{1}{1-\gamma} \log \left[\left(\frac{\alpha \theta}{\beta^{\theta}}\right)^{\gamma}(1+\lambda)^{\gamma} \sum_{i=0}^{\infty} w_{i} \Gamma\left[\frac{1}{\theta}(\theta \gamma-\gamma+1)\right]\right]$

Proof

$$
\begin{aligned}
f^{\gamma}(x) & =\left[\frac{\alpha \theta}{\beta^{\theta}} x^{\theta-1} \exp \left[-\alpha\left(\frac{x}{\beta}\right)^{\theta}\right]\left\{1+\lambda-2 \lambda \exp \left[-\alpha\left(\frac{x}{\beta}\right)^{\theta}\right]\right\}\right]^{\gamma} \\
& =\left(\frac{\alpha \theta}{\beta^{\theta}}\right)^{\gamma}(1+\lambda)^{\gamma} \sum_{i=0}^{\infty}(-1)^{i}\left(\begin{array}{l}
\gamma \\
i
\end{array}\right)\left(\frac{2 \lambda}{1+\lambda}\right)^{i} x^{\gamma(\theta-1)} \exp \left[-\alpha(\gamma+1)\left(\frac{x}{\beta}\right)^{\theta}\right] .
\end{aligned}
$$

Then,

$$
\begin{aligned}
\int_{0}^{\infty} f^{\gamma}(x) & =\left(\frac{\alpha \theta}{\beta^{\theta}}\right)^{\gamma}(1+\lambda)^{\gamma} \sum_{i=0}^{\infty}(-1)^{i}\left(\begin{array}{l}
\gamma \\
i
\end{array}\right)\left(\frac{2 \lambda}{1+\lambda}\right)^{i} \int_{0}^{\infty} x^{\gamma(\theta-1)} \exp \left[-\alpha(\gamma+1)\left(\frac{x}{\beta}\right)^{\theta}\right] d x \\
& =\theta^{-1} \beta^{\gamma(\theta-1)+1}[\alpha(\gamma+i)]^{-\frac{\gamma}{\theta}(\theta-1)-1} \Gamma\left(\frac{1}{\theta}(\theta \gamma-\gamma+1)\right)
\end{aligned}
$$

Substituting equation (15) in equation (14), then

$I_{R}(\gamma)=\frac{1}{1-\gamma} \log \left[\left(\frac{\alpha \theta}{\beta^{\theta}}\right)^{\gamma}(1+\lambda)^{\gamma} \sum_{i=0}^{\infty} w_{i} \Gamma\left(\frac{1}{\theta}(\theta \gamma-\gamma+1)\right)\right]$

where

$w_{i}=w_{i}(\lambda, \gamma)=(-1)^{i}\left(\begin{array}{l}\gamma \\ i\end{array}\right)\left(\frac{2 \lambda}{1+\lambda}\right)^{i} \theta^{-1} \beta^{\gamma(\theta-1)+1}[\alpha(\gamma+i)]^{-\frac{\gamma}{\theta}(\theta-1)-1}$.

\section{Order statistics}

Let $x_{1}, x_{2}, \ldots, x_{n}$ be a random sample from AGT-W distribution such that $x_{1}<x_{2}<\cdots<x_{n}$, the pdf of the $i^{\text {th }}$ order statistics denoted by $f\left(x_{i: n}\right)$ is given by

$$
\begin{aligned}
f\left(x_{i: n}\right) & =\frac{n !}{(i-1) !(n-i) !} f(x) F(x)^{i-1}(1-F(x))^{n-i} \\
& =\frac{n !}{(i-1) !(n-i) !} f(x) \sum_{j=0}^{n-i}(-1)^{j}\left(\begin{array}{c}
n-i \\
j
\end{array}\right) F(x)^{i+j-1} .
\end{aligned}
$$

We have that

$$
\begin{aligned}
& F(x)^{i+j-1}=\left((1+\lambda)\left\{1-\exp \left[-\alpha\left(\frac{x}{\beta}\right)^{\theta}\right]\right\}-\left\{1-\exp \left[-\alpha\left(\frac{x}{\beta}\right)^{\theta}\right]\right\}^{2}\right)^{i+j-1} \\
& F(x)^{i+j-1}=\left((1+\lambda)\left[1-\exp \left(-\alpha\left(\frac{x}{\beta}\right)^{\theta}\right)\right]-\left[1-\exp \left(-\alpha\left(\frac{x}{\beta}\right)^{\theta}\right)\right]^{2}\right]^{i+j-1}
\end{aligned}
$$

Applying the binomial series expansion and simplifying, the pdf of the $i^{\text {th }}$ order statistics of AGT-W is obtained as

$$
\begin{aligned}
f\left(x_{i: n}\right) & =\frac{n !}{(i-1) !(n-i) !}(1+\lambda) \frac{\alpha \theta}{\beta^{\theta}} A x^{\theta-1} \exp \left[-\alpha\left(\frac{x}{\beta}\right)^{\theta}\right] \\
& -\frac{n !}{(i-1) !(n-i) !}(1+\lambda) \frac{\alpha \theta}{\beta^{\theta}} A x^{\theta-1} \exp \left[-\alpha\left(\frac{x}{\beta}\right)^{\theta}\right]
\end{aligned}
$$

where 


$$
A=\sum_{j=0}^{n-i}(-1)^{j}\left(\begin{array}{c}
n-i \\
j
\end{array}\right) \sum_{k, m=0}^{\infty}(-1)^{k+m}(1+\lambda)^{i+j-k-1} \lambda^{k}\left(\begin{array}{c}
i+j-1 \\
k
\end{array}\right)\left(\begin{array}{c}
i+j+k-1 \\
m
\end{array}\right)
$$

This implies that the density function of $i^{\text {th }}$ order statistics of AGT-W distribution is a linear combination of gamma function.

\section{Estimation}

The method of maximum likelihood is used for the estimation of the parameters of the AGT-W $(\theta, \alpha, \beta, \lambda)$ distribution. The likelihood function is given by

$$
\begin{aligned}
l(\Theta)= & n \log (\alpha \theta)-n \theta \log (\beta)+(\theta-1) \sum_{i=1}^{n} \log \left(x_{i}\right)-\alpha \sum_{i=1}^{n}\left(\frac{x_{i}}{\beta}\right)^{\theta} \\
& +\sum_{i=1}^{n} \log \left\{1+\lambda-2 \lambda \exp \left[-\alpha\left(\frac{x_{i}}{\beta}\right)^{\theta}\right]\right\}
\end{aligned}
$$

Where $\Theta=(\theta, \alpha . \beta, \lambda)$ is a vector of parameters for AGT-W distribution.

The maximum likelihood estimators $\hat{\Theta}=(\hat{\theta}, \hat{\alpha}, \hat{\beta}, \hat{\lambda})$ of AGT-W distribution is obtained taking the partial derivative of (18) with respect to $\Theta$, equating to zero and solve for the parameters.

$$
\begin{aligned}
& \frac{\partial l(\Theta)}{\partial \theta}=\frac{n}{\theta}-n \log (\beta)+\sum_{i=1}^{n} \log \left(x_{i}\right)-\alpha \sum_{i=1}^{n}\left(\frac{x_{i}}{\beta}\right)^{\theta} \log \left(\frac{x_{i}}{\beta}\right)+\sum_{i=1}^{n} \frac{2 \lambda \alpha\left(\frac{x_{i}}{\beta}\right) \log \left(\frac{x_{i}}{\beta}\right) \exp \left[-\alpha\left(\frac{x_{i}}{\beta}\right)^{\theta}\right]}{\left\{1+\lambda-2 \lambda \exp \left[-\alpha\left(\frac{x_{i}}{\beta}\right)^{\theta}\right]\right\}}=0 \\
& \frac{\partial l(\Theta)}{\partial \alpha}=\frac{n}{\alpha}-\sum_{i=1}^{n}\left(\frac{x_{i}}{\beta}\right)^{\theta}+\sum_{i=1}^{n} \frac{2 \lambda x^{\theta} \exp \left[-\alpha\left(\frac{x_{i}}{\beta}\right)^{\theta}\right]}{\beta^{\theta}\left\{1+\lambda-2 \lambda \exp \left[-\alpha\left(\frac{x_{i}}{\beta}\right)^{\theta}\right]\right\}}=0 \\
& \frac{\partial l(\Theta)}{\partial \beta}=-\frac{n \theta}{\beta}+\sum_{i=1}^{n} \frac{\alpha \theta}{x_{i}}\left(\frac{x_{i}}{\beta}\right)^{\theta+1}+\sum_{i=1}^{n} \frac{2 \lambda \alpha \theta\left(\frac{x_{i}}{\beta}\right)^{\theta+1} \exp \left[-\alpha\left(\frac{x_{i}}{\beta}\right)^{\theta}\right]}{x_{i}\left\{1+\lambda-2 \lambda \exp \left[-\alpha\left(\frac{x_{i}}{\beta}\right)^{\theta}\right]\right\}}=0 \\
& \frac{\partial l(\Theta)}{\partial \lambda}=\sum_{i=1}^{n} \frac{1-2 \exp \left[-\alpha\left(\frac{x_{i}}{\beta}\right)^{\theta}\right]}{\left\{1+\lambda-2 \lambda \exp \left[-\alpha\left(\frac{x_{i}}{\beta}\right)^{\theta}\right]\right\}}=0
\end{aligned}
$$

Asymptotically, the distribution of the MLE estimators $\hat{\Theta}$ can be approximately multivariate normal. Thus as $\rightarrow \infty$, the asymptotic distribution of the MLE $\hat{\Theta}$ is given by

$$
\left(\begin{array}{l}
\widehat{\theta} \\
\widehat{\alpha} \\
\widehat{\beta} \\
\widehat{\lambda}
\end{array}\right)=N\left[\left(\begin{array}{l}
0 \\
0 \\
0 \\
0
\end{array}\right),\left[\begin{array}{llll}
V_{11} & V_{12} & V_{13} & V_{14} \\
V_{21} & V_{22} & V_{23} & V_{24} \\
V_{31} & V_{32} & V_{33} & V_{34} \\
V_{41} & V_{42} & V_{43} & V_{44}
\end{array}\right]\right]
$$

Where

$$
V_{i j}=-E\left[\begin{array}{llll}
\frac{\partial^{2} l(\Theta)}{\partial \theta^{2}} \frac{\partial^{2} l(\Theta)}{\partial \theta \partial \alpha} & \frac{\partial^{2} l(\Theta)}{\partial \theta \partial \beta} & \frac{\partial^{2} l(\Theta)}{\partial \theta \partial \lambda} \\
\frac{\partial^{2} l(\Theta)}{\partial \alpha \partial \theta} \frac{\partial^{2} l(\Theta)}{\partial \alpha^{2}} & \frac{\partial^{2} l(\Theta)}{\partial \alpha \partial \beta} & \frac{\partial^{2} l(\Theta)}{\partial \alpha \partial \lambda} \\
\frac{\partial^{2} l(\Theta)}{\partial \beta \partial \theta} \frac{\partial^{2} l(\Theta)}{\partial \beta \partial \alpha} & \frac{\partial^{2} l(\Theta)}{\partial \beta^{2}} & \frac{\partial^{2} l(\Theta)}{\partial \partial \beta \lambda} \\
\frac{\partial^{2} l(\Theta)}{\partial \lambda \partial \theta} \frac{\partial^{2} l(\Theta)}{\partial \lambda \partial \alpha} & \frac{\partial^{2} l(\Theta)}{\partial \lambda \partial \beta} & \frac{\partial^{2} l(\Theta)}{\partial \lambda^{2}}
\end{array}\right] ; \mathrm{i}, \mathrm{j}=1,2,3,4
$$

Solving the inverse matrix of the observed information matrix (19) gives the asymptotic variance and co-variance of the MLE $\hat{\theta}, \hat{\alpha}, \widehat{\beta}$ and $\hat{\lambda}$. The $(1-\mathrm{p}) 100 \%$ asymptotic confidence intervals for $\Theta$ are obtained as

$\hat{\theta} \pm \mathrm{Zp}_{2} \sqrt{\widehat{\mathrm{V}}_{11}} ; \widehat{\alpha} \pm \mathrm{Zp} / 2 \sqrt{\widehat{\mathrm{V}}_{22}} ; \widehat{\beta} \pm \mathrm{Zp}_{2} \sqrt{\widehat{\mathrm{V}}_{33}} ; \hat{\lambda} \pm \mathrm{Zp} / 2 \sqrt{\widehat{\mathrm{V}}_{44}}$.

\section{Applications}

In this section, we illustrated the potentials of AGT-W through the application of two real-life data sets. The AGT-W is compared with other competing distributions; Kumaraswamy-Weibull (Kum-W), Beta-Weibull (Beta-W), and Beta-Exponential-Weibull (BetaE-W), and Gamma-Weibull (Gam-W). The maximum likelihood estimates of the parameters of the distributions and its standard errors are obtained. The goodness-of-fits statistics; the log-likelihood $(\ell)$, Akaike information criterion (AIC), Bayesian information criterion (BIC) are also obtained to determine the distribution with a better fit. In general, distribution with the smallest value of the goodness-of-fit statistics has a better fit. The results of the estimates (in parenthesis are the standard errors of the parameters) of fits are shown in table 5 and table 7 
for the first and second data set respectively. Table 6 and Table 8 indicate the goodness-of-fit statistics; AIC and BIC for the first and second data set respectively.

\subsection{Data set 1}

The first data set is on the life fatigue fracture of Kevlar 373/epoxy that were subjected to constant pressure at the $90 \%$ stress level until all had failed, for previous studies with the dataset see [11], [14], [15]. Figure 3 indicates the pdfs and cdf with empirical cdf of the fitted distributions

\subsection{Date set 2}

The second data set is on 346 nicotine measurements made from several brands of cigarettes in 1998 studied by [16]. The plots of the fitted pdfs and cdfs with empirical cdf are shown in Figure 4.

\begin{tabular}{|c|c|c|c|c|c|c|}
\hline Distributions & $\hat{\mathrm{a}}$ & $\widehat{b}$ & $\hat{\lambda}$ & $\widehat{\alpha}$ & $\widehat{\theta}$ & $\widehat{\beta}$ \\
\hline AGT-W & - & - & $0.795(0.213)$ & $0.629(0.126)$ & $1.050(19.419)$ & $0.927(27.249)$ \\
\hline Kum-W & $1.916(2.151)$ & $5.137(21.836)$ & - & - & $0.828(0.843)$ & $4.582(12.289)$ \\
\hline Beta-W & $1.413(0.638)$ & 0.739 (1.633) & - & - & $1.122(0.298)$ & $1.310(2.140)$ \\
\hline BetaE-W & $0.743(1.647)$ & $0.594(16.834)$ & $1.414(0.639)$ & - & $1.121(0.298)$ & $0.826(20.915)$ \\
\hline
\end{tabular}

Table 6: Goodness-of-Fit Statistics of the First Data Set

\begin{tabular}{llll}
\hline Distributions & $\ell$ & $A I C$ & $B I C$ \\
\hline AGT-W & 121.43 & 250.86 & 260.182 \\
Kum-W & 122.86 & 252.13 & 261.452 \\
Beta-W & 122.16 & 252.31 & 261.635 \\
BetaE-W & 122.16 & 254.31 & 265.966 \\
\hline
\end{tabular}

Table 7: Parameter Estimates of the Second Data Set (Standard Error in Parenthesis)

\begin{tabular}{|c|c|c|c|c|c|c|}
\hline Distributions & $\hat{a}$ & $\widehat{b}$ & $\hat{\lambda}$ & $\widehat{\alpha}$ & $\hat{\theta}$ & $\widehat{\beta}$ \\
\hline AGT-W & - & - & 1.555 (25.506) & $2.295(0.153)$ & $0.588(0.142)$ & $1.015(7.255)$ \\
\hline Gam-W & $02.4353(0.5948)$ & - & - & - & $0.925(0.185)$ & $0.734(0.157)$ \\
\hline Beta-W & $0.7484(0.0732)$ & $0.219(0.016)$ & - & - & $2.962(0.004)$ & $0.599(0.006)$ \\
\hline BetaE-W & $0.7047(0.150)$ & $0.980(60.77)$ & $1.560(37.62)$ & - & $3.325(0.461)$ & $2.492(18.941)$ \\
\hline
\end{tabular}

Table 8: Goodness of Fit Statistics of the Second Data Set

\begin{tabular}{llll}
\hline Distributions & $\ell$ & $A I C$ & \\
\hline$A G T-W$ & 92.892 & 193.785 & 208.266 \\
Gam-W & 95.299 & 196.599 & 208.460 \\
Beta-W & 94.042 & 196.085 & 210.567 \\
BetaE-W & 94.961 & 199.922 & 218.024 \\
\hline
\end{tabular}

Estimated pdf's

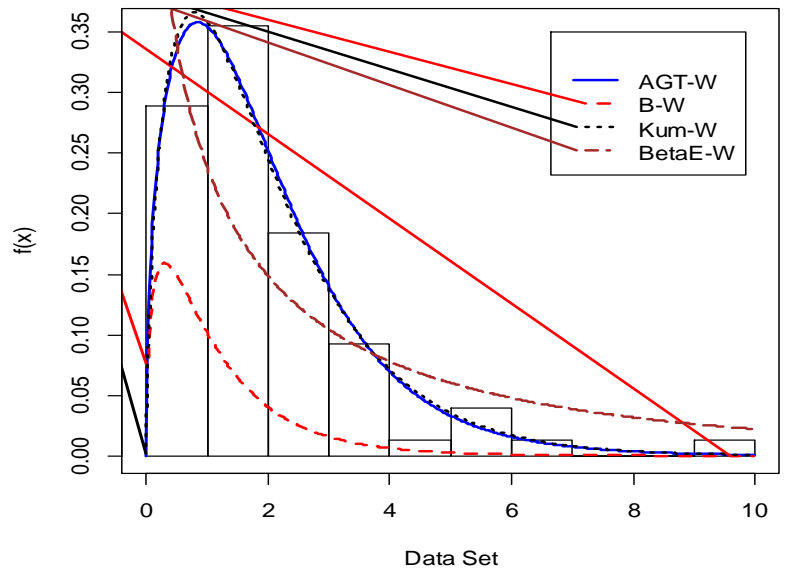

Estimated cdf's

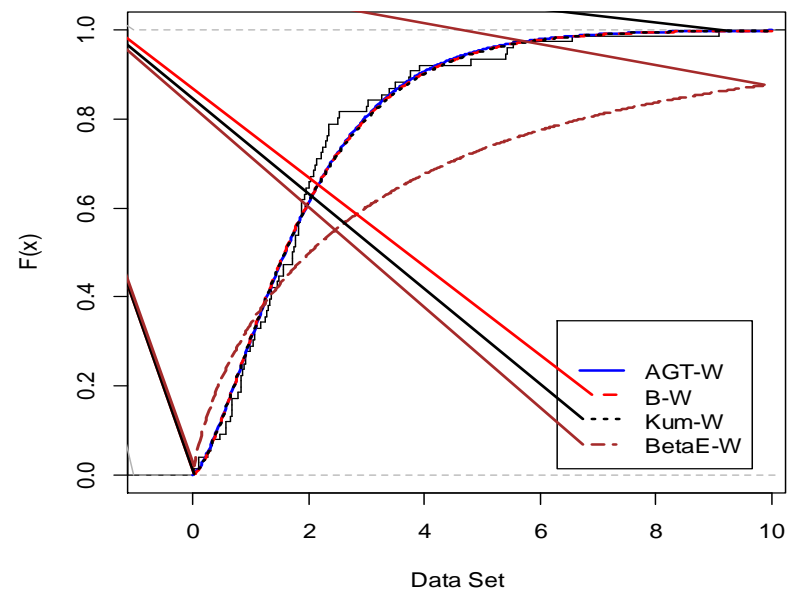

Fig. 3: Plot of Estimated Pdf and Cdf with Empirical Cdf of the First Data Set. 
Estimated pdf's

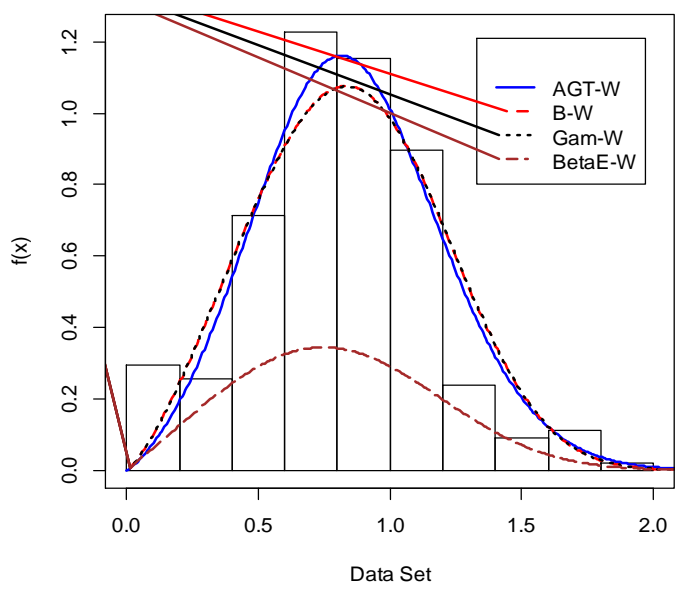

Estimated cdf's

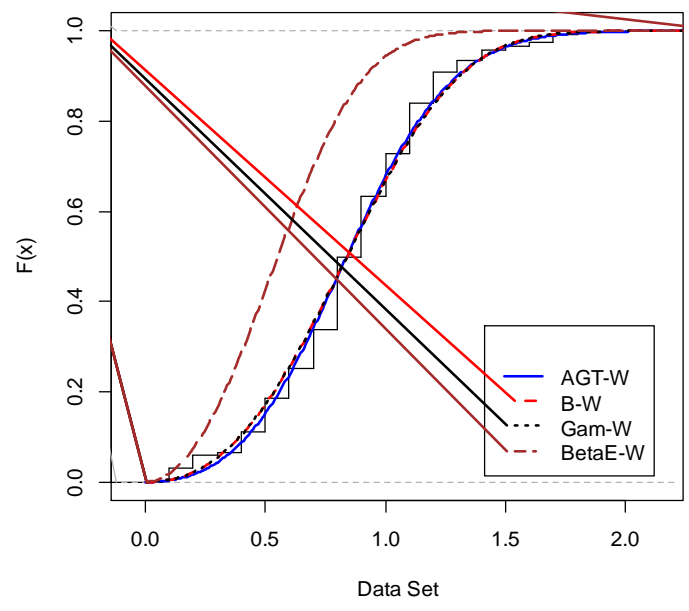

Fig. 4: Plots of Estimated PDFS and CDFS with Empirical CDF of the Second Data Set

\section{Conclusion}

We studied the some mathematical characterizations including the shape, hazard rate function, non-central moment, mgf, entropy, and order statistic of four-parameter AGT-W distribution. The estimation of the unknown parameters of the new distribution was done using the maximum likelihood approach. The potential and flexibility of AGT-W were illustrated with two real-life data sets and comparison based on goodness-of-fit statistics with other existing distributions in the statistical literature was also done. The results indicate that the goodness-of-fit statistics of AGT-W are the smallest among the competing distributions. This implies that AGT-Weibull distribution provided a better fit. We hope that the distribution will find a wider application in other areas such as biology, finance, engineering etc.

\section{References}

[1] G.S. Mudholkar and D.K. Saivastava. Exponentiated Weibull family for analyzing bathtub failure-rate data. IEEE Transactions on Reliability, 42, 2, 1993, 299-302. https://doi.org/10.1109/24.229504.

[2] G.S. Mudholkar, D.K. Saivastava and M. Freimer. Exponentiated Weibull Family: A reanalysis of the bus-motor-failure data. Technometrics, 37,4, 1995, 436-445. https://doi.org/10.1080/00401706.1995.10484376.

[3] G.S. Mudholkar, D.K. Saivastava and G.D. Kollia. A generalization of the weibull distribution with application to the analysis of survival data. Journal of the American statistical association, 91, 436, 1996, 1575-1583. https://doi.org/10.1080/01621459.1996.10476725.

[4] M.E. Ghitany, E.K. Al-Hussaini and R.A. Al-Jarallah. Marshall-Olkin extended weibull distribution and its application to censored data. Journal of Applied Statistics, 32, 10, 2005, 1025-1034. https://doi.org/10.1080/02664760500165008.

[5] C. Lee, F. Famoye and O. Olumolade. Beta-Weibull Distribution: Some Properties and Applications to censored Data. Journal of Modern Applied Statistical Methods, 6, 2007, 173-186. https://doi.org/10.22237/jmasm/1177992960.

[6] G.O. Silva, E.M.M. Ortega and G.M. Cordeiro. The beta modified Weibull distribution. Life Data Anal, 16, 2010, 409-430. https://doi.org/10.1007/s10985-010-9161-1.

[7] G.M. Cordeiro, E.M.M. Ortega and S. Nadarajah. The Kumaraswamy Weibull distribution with application to failure data. Journal of the Franklin Institution, 347, 2010, 1399-1429. https://doi.org/10.1016/j.jfranklin.2010.06.010.

[8] G.R. Aryal and C.P. Tsokos. Transmuted Weibul distribution: A generalization of the Weibull Probability distribution. European Journal of Pure and Applied Mathematics, 4, 2, 2011, 89-102.

[9] R. Al-Aqtash, C. Lee, and F. Famoye. Gumbel-Weibull Distribution:Properties and Applications. Journal of Modern Applied Statistical Methods, 13, 2014, 201-225. https://doi.org/10.22237/jmasm/1414815000.

[10] G.M. Cordeiro, E.M.M. Ortega, and G. Silva. The Beta extended Weibull family. Journal of Probability and Statistical Science, 10, $2012,15-40$.

[11] F. Merovci, M. Alizadeh and G.G. Hamedani. Another generalized transmuted family of distributions: Properties and applications. Austrian Journal of Statistics, 45, 2016, 71-93. https://doi.org/10.17713/ajs.v45i3.109.

[12] L. N. Johnson, S. Kotz and N. Balakrishnan. Continuous Univariate distributions, Volume1 Second Edition. John Wiley \& Sons Inc, 1995.

[13] A. Renyi. On Measures of Entropy and Information, Proceedings of the Fourth Berkeley Symposium on Mathematical Statistics and Probability, Volume 1: Contributions to the Theory of Statistics, 547--561, University of California Press, Berkeley, Calif, 1961.

[14] DF Andrews and AM Herzberg. A collection of problems from many fields for the student and research worker, New York: Springer-verlag, 1985.

[15] R.E. Barlow, R.H. Toland, and T. Freeman. (1984). A Bayesian analysis of stress-rupture life of Kevlar 49/epoxy spherical pressure vessels. In T. D. Dwivedi (Ed.), Proceedings of the Canadian Conference in Applied Statistics. New York:Marcel Dekker, 1984.

[16] L. Handique, S. Chakraborty and G.G. Hamedani. The Marshall-Olkin Kumarawamy-G family of distributions. Journal of Statistical Theory and Applications, 16, 2017, 427-447. https://doi.org/10.2991/jsta.2017.16.4.2. 